\title{
Effect of Specific Nutrients on Ovulation, Oocytes Development, Gene Expression and Coupling Success in Mice
}

\author{
P. Vitullo, C. Cossetti, M. A. Virmani* \\ Research, Innovation and Development, Sigma-Tau Health Science International BV, Utrecht, The Netherland \\ Email: ${ }^{\star}$ ashraf.virmani@alfasigma.com
}

How to cite this paper: Vitullo, P., Cossetti, C. and Virmani, M.A. (2018) Effect of Specific Nutrients on Ovulation, Oocytes Development, Gene Expression and Coupling Success in Mice. International Journal of Clinical Medicine, 9, 660-674. https://doi.org/10.4236/ijcm.2018.99055

Received: August 14, 2018

Accepted: September 11, 2018

Published: September 14, 2018

Copyright (c) 2018 by authors and Scientific Research Publishing Inc. This work is licensed under the Creative Commons Attribution International License (CC BY 4.0).

http://creativecommons.org/licenses/by/4.0/ (c) (i) Open Access

\begin{abstract}
Introduction: The roles of genetic, epigenetic, metabolic and other environmental factors such as nutrition and stress, are becoming evident for a successful and healthy pregnancy. This raises the possibility and question, if and how, we improve the probability of pregnancy and of a healthy fetus? The present study examined the role of metabolic, antioxidant and minerals and the results suggest that these factors may positively influence the oocyte quality and the pregnancy rate. Methods: CD1 female mice aged 15 and 5 weeks were divided into four groups of ten each and treated by intragastric gavage daily for 3 weeks. G1: Vehicle; G2: Carnitines (L-carnitine $0.4 \mathrm{mg}$ and acetyl-L-carnitine $0.12 \mathrm{mg} / \mathrm{mouse}$ ); G3: Microelements (Zinc $4 \mathrm{ng}$, Copper $0.8 \mathrm{ng}$, Iron $7 \mathrm{ng} /$ mouse); G4: G3+G2. At the end of the treatment period superovulation was induced and oocytes were collected to assess their quantity and quality. Further, in vitro fertilization (IVF) experiments were performed to assess the preimplantation embryo development. The birth success rate was also analyzed in old and young female. The mice were in vivo fertilized. qRT-PCR were performed to analyze a possible modulation in key genes of the reproductive process. Results: The number of oocytes was significantly higher in groups 2 and 4 compared to the control group. The oocyte number in group 3 was not affected. The level of degraded oocytes was $29.1 \%$ and 19.3\% (group 2 and 4) versus 34.3\% (control). Concomitantly, the numbers of embryos arriving to successful birth were also increased in G4, both in the old and young group of mice. Preliminary analysis of genes affected evidenced that AMH was up regulated in the ovary and KITL in the uterus in group 2. Conclusion: Results showed that L-carnitine, acetyl-L-carnitine and micronutrients were able to improve both oocytes quality and success rate of pregnancy. Further studies are planned to further examine ways to improve pregnancy and fetal health.
\end{abstract}




\section{Keywords}

Pregnancy, L-Carnitine, Acetyl-L-Carnitine, Micronutrients, Oocytes, Birth, Fetal Health, Embryos, Key Genes, Fertilization, Reproductive

\section{Introduction}

Genes and cellular metabolism are intrinsically linked to fertility and successful pregnancy. It is estimated that 10 to 15 percent of couples have trouble getting pregnant or getting to a successful delivery, in the USA according to the Centers for Disease Control and Prevention (CDC). The infertility results due to issues with the female about one-third of the time and due to male infertility factors about one-third of the time. In the rest, the cause is either unknown or a combination of male and female factors. As stated by the National Center for Health Statistics (NCHS), the fertility rates in the US have declined over the last few decades and many couples have trouble conceiving. The reasons for the declines are not completely understood but are thought to be related to a number of factors such as nutrition, pollution, stress, and general lifestyle issues. Women who experience problems achieving a recognized conception also have elevated rates of early unrecognized pregnancy loss [1] and elevated rates of clinical spontaneous abortion [2]. Indeed, the recent review by Gray and Becker [2] suggests that delayed conception and the early pregnancy loss may share a common etiology, possibly through events or exposures prior to or during implantation and embryogenesis. The decline in fertility could in part be explained by a decrease in the ovum quality, as well as the physiological processes involved in female fertility. At birth, there are approximately one million eggs; and by the time of puberty, only about 300,000 remain. Of these only about 300 to 400 will be ovulated during a woman's reproductive lifetime, the question of how to improve egg quality is more recent. There is growing consensus that egg quality declines with age. This could be connected to the accumulation of cellular damage with time, a reduction in cellular metabolic processes, which in turn leads to a reduction in energy production and in increase in cellular oxidative stress, which can trigger abnormal physiological processes especially immune and inflammation processes.

\subsection{Role of Cellular and Mitochondrial Metabolism in Fertility}

The majority of cellular energy is produced in the mitochondria, which are specialized organelles found in the cytoplasm of the cells. The impairment of cytoplasmic and in particular the mitochondrial function would lead to a drastic reduction in cellular energy and an increased oxidative stress. The escape of reactive oxygen species (ROS) from the mitochondria could trigger a number of processes such as inflammation that in turn play a key role in initiating pathology. Damage or deficiency in appropriate metabolic cofactors and substrates to 
mitochondria results in aberrant functionality of the respiratory chain and oxidative phosphorylation resulting in reduced energy and increased cytotoxicity. Free radical production also induces inflammatory processes due to the misfolding of proteins and other mechanisms. The use of antioxidant substances able to reduce or quench ROS may thus counteract cellular damage. Research into mitochondriotropic agents such as carnitines, coenzyme Q10 and nicotinamide have shown potential to improve various factors associated with body energy, hormonal regulation, uterus weight as well as possible stabilization of cellular membranes and the genome [3]. The role of these compounds in epigenetics and effects upon genomic stability per se is also becoming important in fertility [4]. L-Carnitine, which is essential in fatty acid metabolism, has been shown to prevent mitochondrial damage induced in the rat choroid plexus by medium chain fatty acids [5] or by mitochondrial toxins [6].

\subsection{The Emerging Role of Nutrigenomics, Metabolomics and Oxidative Stress in Fertility}

Many recent studies have shown that some biochemical and genetic alterations occur in the oocyte and take part in the natural history of female infertility [7] [8] [9]. Any dysfunction in the genes and therefore in the related protein, for example an enzyme protecting against reactive oxygen species (ROS) called superoxide dismutase I (SOD) is known to lead to female folliculogenesis defects and cause a failure in maintaining pregnancy.

Studies suggest that oxidative stress is associated with decreased female fertility in animals and in-vitro models; epidemiological evidences strongly suggest that exposure to oxidative stress can influence the timing and maintenance of a viable pregnancy (e.g. preeclampsia).

The identification and evaluation of appropriate genetic, metabolic and biochemical markers correlating with early steps of disease onset and progression can be used as a key in preventing this type of dysfunction.

\subsection{Carnitines and Female Fertility}

Carnitine is a natural product synthesized in mammals from the essential amino acids lysine and methionine or obtained from dietary sources (Figure 1).

It is essential in fatty acid metabolism and studies are showing its role in the epigenetic modulation of cellular functions. Carnitine is necessary for cellular energy production and its dysfunction results in various diseases. These are related to abnormal mitochondrial function and thus reduced energy production and increased oxidative stress. Growing evidence supports the hypothesis of the control of oxidative stress by metabolism. Carnitine plays an antioxidant role controlling ROS pathway, showing specific preventive action against lipid peroxidation and membrane damage. These represent some molecular events that culminate in the initiation of a disease process. Studies suggest that carnitine reduces oocyte cytoskeletal damage and embryo apoptosis [10], furthermore it may also stabilize the hypothalamic pituitary gonadal axis [6]-[11]. Carnitines 
<smiles>C[N+](C)(C)C[C@H](O)CC(=O)O</smiles>

L-Carnitine<smiles>CC(=O)O[C@@H](CC(=O)O)C[N+](C)(C)C</smiles>

Acetyl-L-Carnitine

Figure 1. Chemical structures of L-carnitine and acetyl-L-carnitine.

play a central role in fatty acid beta oxidation, that is involved in the acquisition of oocyte competence during which specific enzymes are expressed [12]. Studies show a correlation between the levels of CPT2 expression and embryo developmental competence [13]. Indeed, the inhibition of beta-oxidation during oocyte maturation or zygote division impairs subsequent blastocyst development. In contrast, L-carnitine supplementation during oocyte maturation significantly increases beta-oxidation and improves developmental competence [14]. In addition, its acetylated form, acetyl-L-carnitine (ALC), protects cells exposed to neurotoxins and mitochondrial inhibitors [6]. Thus, together with antioxidant compounds like Q10 a protective action may be hypothesized on ovum and other tissues vital for successful fertility.

However, the quality of the ovum and the state of the uterus is also very important for a successful pregnancy. There is growing consensus that egg quality declines with age and in conditions of abnormal energy balance, such as in polycystic ovary syndrome (PCOS). Indeed, what remain to be elucidated is the critical period between ovulation and implantation. During this period the successfully fertilized egg has to rely exclusively on its own energy reserves to start growing form the zygote to the blastocysts stage. The energy during this period comes from the mitochondria of the oocyte; if this energy runs out the egg stops dividing and implantation is not achieved. This would result in pregnancy loss. In the $70 \%$ of PCOS affected women (coupled or not with insulin resistance) inhibition of ovulation, impaired of maturation of viable egg, and reduced egg quality and implantation rate, can occur. It is possible that improving the energy balance before the ovulation process would improve the success of pregnancy. Further the oocyte quality could be improved by substances such as carnitines and antioxidant compounds, increasing the chances of a successful pregnancy. All these factors are becoming important in the treatment of female infertility.

\section{Material and Methods}

\subsection{Ethics Statement}

All animal procedures were conducted under the approval of the Italian National 
Institute of Health. Animal care was conformed to the European Council Directive 86/609/EEC and all experiments including animals were approved by the review board of the Italian National Institute of Health (Istituto Superiore di Sanita', ISS) and authorized by the Italian Ministry of Health. Animals were sacrificed by cervical dislocation. All efforts were made to minimize suffering.

\subsection{The Experiments Were Conducted in Three Steps}

- First step: Female 8 weeks old CD1 mice were divided into four groups of ten each and treated daily for 3 weeks by intragastrical gavage. G1: Vehicle; G2: Carnitines (L-carnitine $0.4 \mathrm{mg}$ and acetyl-L-carnitine $0.12 \mathrm{mg} / \mathrm{mouse}$ ); G3: microelements (Zinc 4 ng, Copper 0.8 ng, Iron 7 ng/mouse); G4: G3+G2. The treatment dose for the mice was calculated approximately based on the recommended healthy dietary allowances for human consumption. After treatment superovulation was induced, oocyte collected to assess quality and quantity. Moreover, in vitro fertilization (IVF) experiments were performed to evaluate the preimplantation embryos development [15]. Details for superovulation, oocyte collection and IVF are given below (Figure 2).

- Second step: The ovary of the mice were surgically explanted and the RNA extracted, and expression of AMH, FSHB, CEPBP, LHR, and CDX2 genes was tested, being them related to ovary aging and minor production of oocytes (CEPBP, LHR and CDX2), to ovarian reserve and stimulation of oocytes production (AMH and FSHB). RNA was extracted and gene expression evaluated by Reverse transcriptase PCR (RT-PCR) and quantitative real-time PCR as described below.

- Third step: Female 15 and 5 weeks old CD1 mice were divided into four groups of ten each and treated daily for 3 weeks by intragastric gavage. G1: Vehicle; G2: Carnitines (L-carnitine $0.4 \mathrm{mg}$ and acetyl-L-carnitine 0.12 mg/mouse); G3: microelements (Zinc 4 ng, Copper 0.8 ng, Iron 7 ng/mouse); G4: G3+G2. To assess the successful rate of birth in old and young female, mice were in vivo fertilized, as described below.

\begin{tabular}{|c|c|c|c|}
\hline & \multicolumn{2}{|c|}{$\begin{array}{l}3 \text { WEEKS OF TREATMENT } \\
\text { (intragastric administration) }\end{array}$} & $\begin{array}{l}\text { NUTRIENTS } \\
\text { (per mouse) }\end{array}$ \\
\hline & 1 & CTRL & Vehicle \\
\hline \multirow{3}{*}{$\begin{array}{l}\text { Female } 15 \text { and } 5 \text { weeks } \\
\text { old CD1 mice } \\
\text { each group } N=10\end{array}$} & 2 & Carnitines & $\begin{array}{c}\text { L-Carnitine } 0.4 \mathrm{mg} \\
\text { Acetyl-L-Carnitine } 0.12 \mathrm{mg}\end{array}$ \\
\hline & 3 & Zinc, Copper, Iron & $\begin{array}{l}\text { Zinc } 4 \mathrm{ng} \\
\text { Copper } 0.8 \mathrm{ng} \\
\text { Iron } 7 \mathrm{ng}\end{array}$ \\
\hline & 4 & $\begin{array}{c}2+3 \\
\text { (Carnitines }+ \text { Zinc, Copper, } \\
\text { Iron) }\end{array}$ & \\
\hline
\end{tabular}

Figure 2. Methods. 


\subsection{Collection of Organs, Spermatozoa and Oocytes}

Germ free CD1 female and male mice were purchased from Charles River (Calco, Italy). Viable somatic cell-free spermatozoa were obtained by puncturing cauda epididymis with a needle and collected after a 30 - 60 min "swim-up" selection step in FM medium [16] to perform IVF experiments. Oocytes for IVF experiment were obtained by surgical resection of ovaries as well as preimplantation embryos, obtained by natural breeding [16]. Surgically uterus and ovaries obtained were also stored in Trizol reagent (Invitrogen) for subsequent RNA extraction.

\subsection{RNA Extraction}

Organs from mice were lysed in Trizol reagent (Invitrogen) and total RNA was extracted according to manufacturer's protocol, without further modification, except for the final step including two $\mathrm{EtOH} 75 \%$ washes rather than one.

\subsection{Reverse Transcriptase PCR (RT-PCR) and Quantitative Real-Time PCR}

$2 \mu \mathrm{g}$ of total RNA extracted was reverse transcribed into cDNA using the iScript Advanced cDNA Synthesis kit for RT-qPCR (Bio-Rad). 200 ng of cDNA were qPCR amplified with specific primer pairs listed below:

AMH: For 5'-gtgagaggagaggggaacac-3', Rev: 5'-gttctccagtctcccctagc-3';

CDX2: For 5'-ctgtcccttccctcgtcttt-3', Rev: 5'-aactgtgttcggatcccctt-3'

CEBPB: For 5'-tgcggggttgttgatgtttt-3', Rev: 5'-tgctcgaaacggaaaaggtt-3';

FSHb: For 5'-tcgtctgccttttagagcca-3', Rev: 5'-ttcctcagccagcttcatca-3'

LHR: For 5'-acccggtgcttttacaaacc-3', Rev: 5'-cgtcgtcccattgaatgcat-3'

GAPDH: For 5'-accacagtccatgccatcac-3', Rev: 5-tccaccaccctgttgctgta-3'

Quantitative real time PCR (qPCR) was performed using SsoAdvanced Universal SYBR Green Supermix (Bio-Rad) following the manufacturer's instructions in a 7500 Fast Real-Time PCR System (Applied Biosystems) under following conditions: one cycle of $95^{\circ} \mathrm{C}$ for $30 \mathrm{~s}, 40$ cycles of $98^{\circ} \mathrm{C}$ for $15 \mathrm{~s}, 60^{\circ} \mathrm{C}$ for 1 min and the instrument default settings for the Melt-Curve analysis. The annealing of the primers was at $60^{\circ} \mathrm{C}$. RNA levels were normalized to the level of GAPDH and calculated as delta-delta threshold cycle $(\Delta \Delta \mathrm{CT})$ [17]. All PCR experiments were performed in triplicate and the results were analyzed with qPCR 7500 Software Download v. 2.0.6.

\subsection{Treatment and Induction of Superovulation in Mice}

At the end of the relevant treatments, in the female 8 weeks old CD1 mice belonging to all the four groups, superovulation was induced by intraperitoneal injection (i.p.) of $5 \mathrm{IU}$ of pregnant mare serum gonadotropin (PMSG) (Folligon, Intervet) followed by $5 \mathrm{IU}$ of human chorionic gonadotropin (hCG) (Corulon, Intervet) after 48 hours. Mice of young group were sacrificed and oocytes collected to assess quantity for each group and to perform in vitro fertilization. 


\subsection{In Vitro Fertilization}

12 - 13 hours after hCG injection, female 8 weeks old CD1 mice were sacrificed; oviducts were removed and squeezed in $1 \mathrm{ml}$ of FM medium. Aliquots of $(1-2)$ $\times 10^{6}$ spermatozoa were withdrawn and added to the egg-containing dishes. Dishes containing both sperm cells and eggs were incubated for 6 hours at $37^{\circ} \mathrm{C}$ with $5.3 \% \mathrm{CO}_{2}$. Non-degraded eggs were then transferred to dishes containing 1 $\mathrm{ml}$ of fresh M-16 medium (Sigma) supplemented with BSA (4 mg/ml), overlaid with mineral oil and further cultured for 7 hours at $37^{\circ} \mathrm{C}$ with $5.3 \% \mathrm{CO}_{2}$. After $24 \mathrm{hr}$ fresh medium was replaced. Embryos were allowed to grow for 5 days up to the blastocyst stage and, on the basis of morphology, the number of degraded and normal developing embryos was assessed under an inverted photomicroscope stage by stage.

\subsection{In Vivo Fertilization}

12 - 13 hours after hCG injection, female 12 and 5 weeks old CD1 mice were mated with males of the same strain. The presence of a vaginal plug on the following morning indicated successful mating. Developmental progression was followed up till the naturally birth of pups.

\subsection{Statistical Analysis}

All the data were descriptively analyzed and presented as $\mathrm{n}$, mean and standard deviation in Table 1 and as mean in Table 2. The figures were created using the data included in the tables. The $\mathrm{p}$-values were derived from the $\mathrm{t}$-test comparing each group with the control one.

\section{Results}

\subsection{Carnitine Treatment Improve Quality and Quantity of Oocyte}

The results of the study that were in part already published [14] and show that the mean number of oocytes/mouse was higherin the groups 2 (carnitines) and 4 (microelements plus carnitines): $32.5(\mathrm{p}=0.273)$ and $31.3(\mathrm{p}=0.378)$ respectively, versus control group: 24.5 (Figure 3 and Table 1).

The number of oocytes in group 3 was not significantly affected (27.2). Furthermore, the number of degraded oocytes in these groups was positively regulated: $27.8 \%(\mathrm{p}=0.163)$ and $19.3 \%(\mathrm{p}=0.004)$ (group 2 and 4 respectively) versus $34.3 \%$ (control group) (Figure 4 and Table 1 ).

This is an important result that showed a significant effect of microelements and carnitines to increase oocytes number and also to maintain their good quality. It is interesting to note, that, as shown in the same figure in the microelements only (Group 3) a higher percentage of degraded oocytes (35.3\%) was observed and this was markedly decreased by the addition of the carnitines (19.3\%), suggesting the positive effect of the combination of both classes of compounds. These observations suggest that the carnitine group seems to give some benefits in both genesis and ovulation process. 
Table 1. Summary of relevant results.

\begin{tabular}{|c|c|c|c|c|}
\hline Parameter (u.m.) & Control & Group 2 & Group 3 & Group 4 \\
\hline \multicolumn{5}{|l|}{ Oocytes } \\
\hline $\mathrm{N}$ & 10 & 9 & 5 & 5 \\
\hline Mean & 24.5 & 32.5 & 27.2 & 31.3 \\
\hline SD & 13.8 & 16.8 & 10.7 & 13.1 \\
\hline p-value ${ }^{*}$ & & 0.273 & 0.712 & 0.378 \\
\hline \multicolumn{5}{|c|}{ CD1 mouse oocyte percentage degraded } \\
\hline $\mathrm{N}$ & 10 & 10 & 10 & 10 \\
\hline Mean & 34.3 & 27.8 & 35.3 & 19.3 \\
\hline $\mathrm{SD}^{* *}$ & 10 & 10 & 10 & 10 \\
\hline p-value ${ }^{*}$ & & 0.163 & 0.826 & 0.004 \\
\hline \multicolumn{5}{|l|}{ 2-cells stage embryos } \\
\hline $\mathrm{N}$ & 10 & 10 & 10 & 10 \\
\hline Mean & 79.2 & 35.1 & 30.9 & 45.2 \\
\hline $\mathrm{SD}^{* *}$ & 30 & 10 & 10 & 20 \\
\hline p-value ${ }^{*}$ & & $>0.001$ & $>0.001$ & 0.008 \\
\hline \multicolumn{5}{|c|}{ Blastocystis stage embryos vs. 2-cells stage } \\
\hline $\mathrm{N}$ & 10 & 10 & 10 & 10 \\
\hline Mean & 59.6 & 86.6 & 16.0 & 18.0 \\
\hline SD & 15 & 20 & 8 & 8 \\
\hline p-value ${ }^{*}$ & & 0.003 & $>0.001$ & $>0.001$ \\
\hline \multicolumn{5}{|c|}{ Pregnancy Rate Old mice } \\
\hline $\mathrm{N}$ & 10 & 10 & 10 & 10 \\
\hline Mean & 70 & 80 & 30 & 60 \\
\hline $\mathrm{SD}^{* *}$ & 4 & 4 & NA & 4 \\
\hline \multicolumn{5}{|c|}{ Pregnancy Rate Young mice } \\
\hline $\mathrm{N}$ & 10 & 10 & 10 & 10 \\
\hline Mean & 90 & 100 & 80 & 100 \\
\hline $\mathrm{SD}^{* *}$ & 4 & 4 & NA & 4 \\
\hline
\end{tabular}

${ }^{*} \mathrm{p}$-values were derived from $\mathrm{t}$-test on each group vs. control group; ${ }^{* *}$ estimated.

\subsection{Carnitine Treatment Positively Affect Preimplantation Development}

The results of IVF experiments performed to establish the magnitude of fertilized eggs and developed embryos, summarized in (Figure 5 and Table 1), showed that despite the treated groups had a minor percentage of 2-cells embryos versus control group, the major part of them reach the blastocyst stage in Group 2 (86.6\% versus 59.6\%) (Figure 6 and Table 1). 
Table 2. Summary of CEBP, LHR and CDX2 gene expression by group.

\begin{tabular}{ccccc}
\hline Parameters & Control & Group 2 & Group 3 & Group 4 \\
\hline $\begin{array}{c}\text { Stimulation of oocytes production } \\
\text { AMH }\end{array}$ & 1 & 2.278 & & \\
$\begin{array}{c}\text { Stimulation of oocytes production } \\
\text { FSHB }\end{array}$ & 1 & 1.264 & & \\
$\begin{array}{c}\text { Gene Analysis } \\
\text { CEBPB }\end{array}$ & 1 & 0.586 & 0.7804 & 0.7006 \\
$\begin{array}{c}\text { Gene Analysis } \\
\text { LHR } \\
\text { Gene Analysis } \\
\text { CDX2 }\end{array}$ & 1 & 0.7766 & 1.6521 & 0.0156 \\
& 1 & 0.8876 & 0.5211 & 0.4408 \\
\hline
\end{tabular}

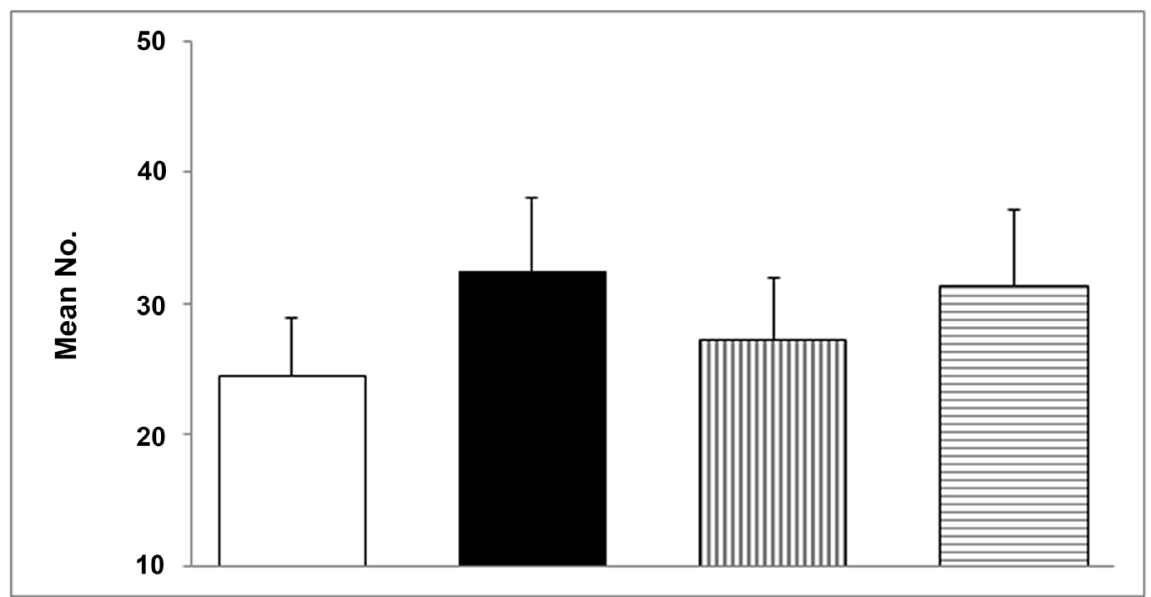

Figure 3. Mean of oocytes.

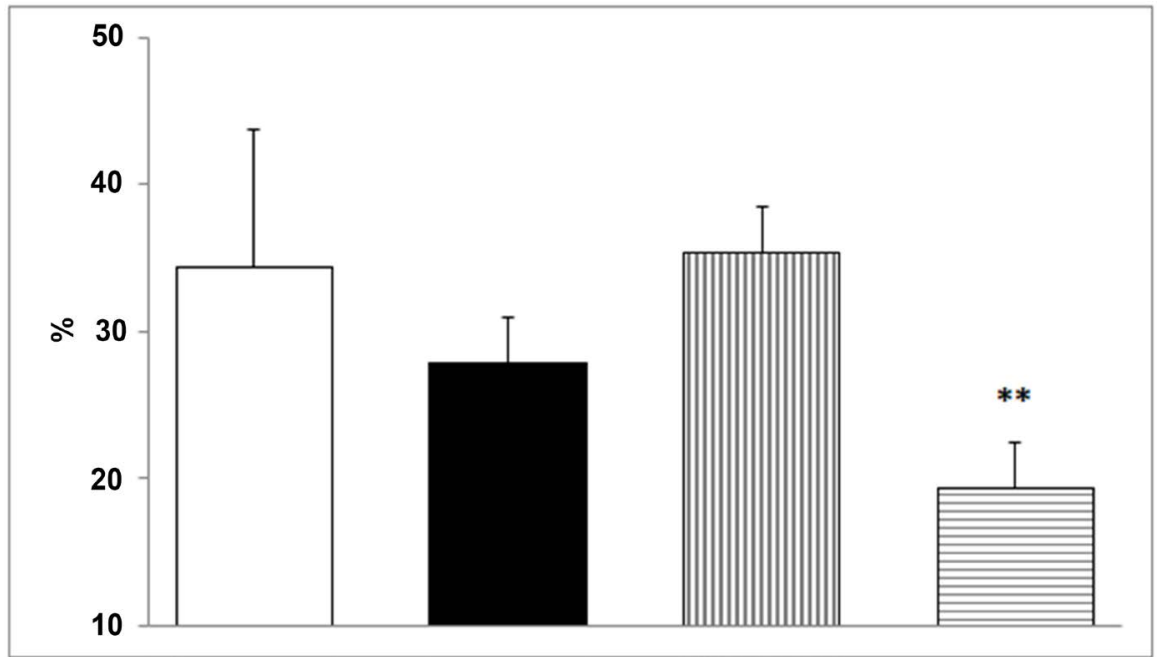

Figure 4. CD1 mouse oocyte percentage degraded.

These results show that carnitines have an important effect on embryo development on 8 weeks mice. 


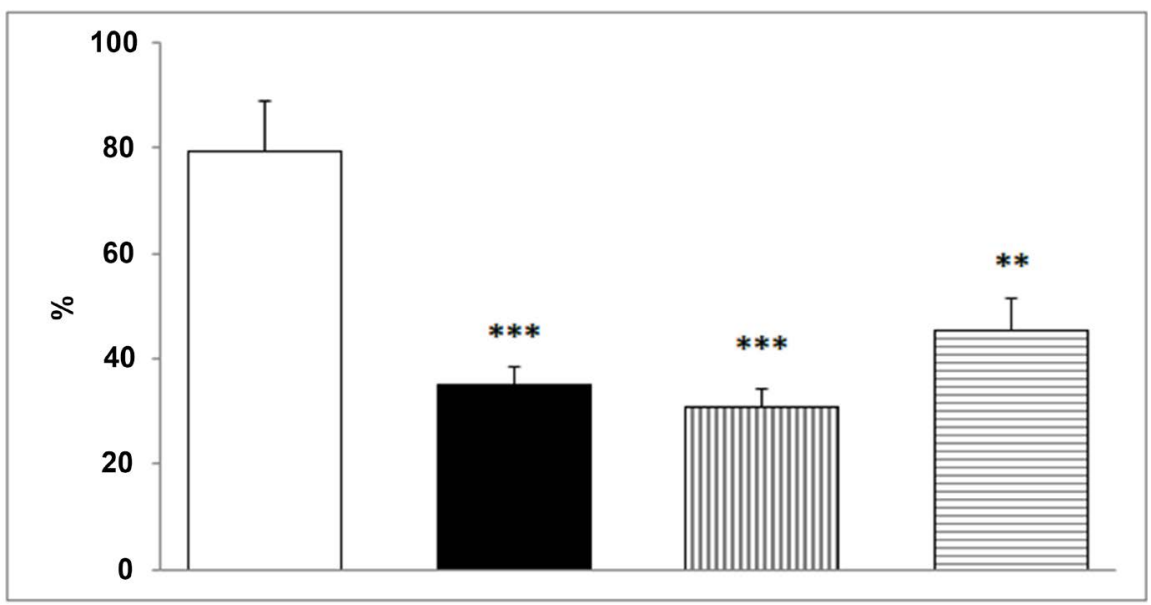

Figure 5. \% 2-cells stage embryos.

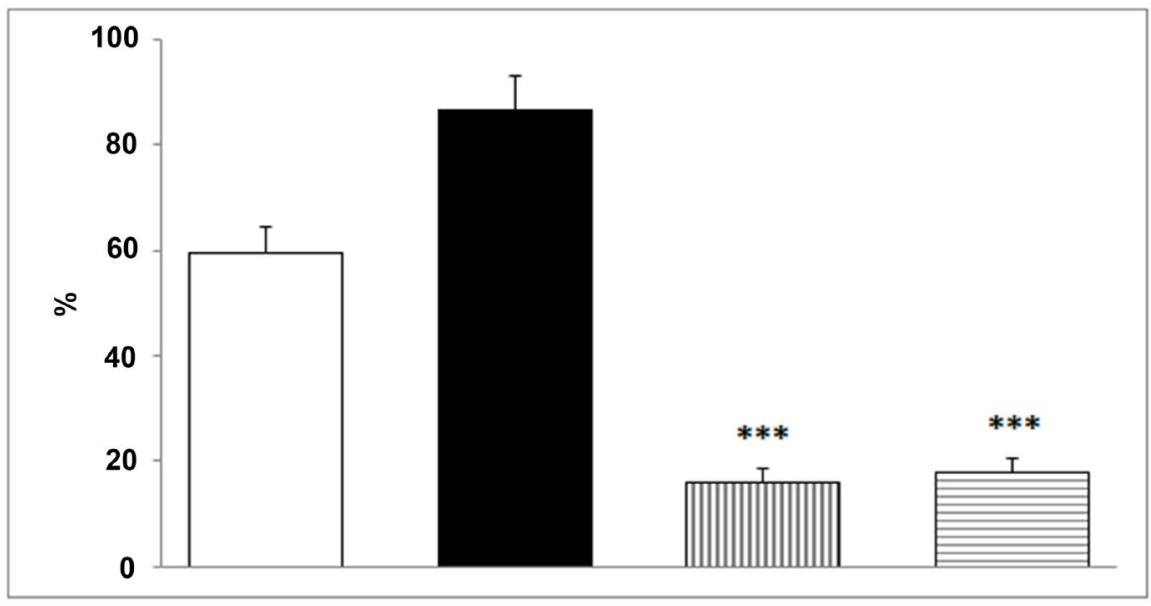

Figure 6. \% Blastocysts stage embryos vs. 2-cells stage.

\subsection{Gene Expression Analysis after Carnitine Treatment}

After treatment, we decided to explore the possible effect of the different supplementations on the gene expression in ovary. Particularly we focused on CEPBP, LHR and CDX2 whose expression is related to ovary aging and minor production of oocytes, $\mathrm{AMH}$ and $\mathrm{FSHB}$, influencing the ovarian reserve and stimulation of oocytes production.

Preliminary analysis of genes affected in these processes showed some interesting trends. The expression levels of AMH and FSHB was upregulated in group 2, the expression of AMH was more than 2 times higher than the control group, FSHB was slightly over expressed (Figure 7 and Table 2). In group 3 and 4 no significant effect on AMH or FSHB expression was evidenced.

The different behavior of CEBP, LHR and CDX2 gene expression was observed in the four groups; in particular, CEBP expression was down regulated 0.6 times compared to control in group 2, the down regulation was still evident, even if to minor extend, in group 3, about 0.8 times the control, and group 4, about 0.7 times of the control (Figure 8 and Table 2). 


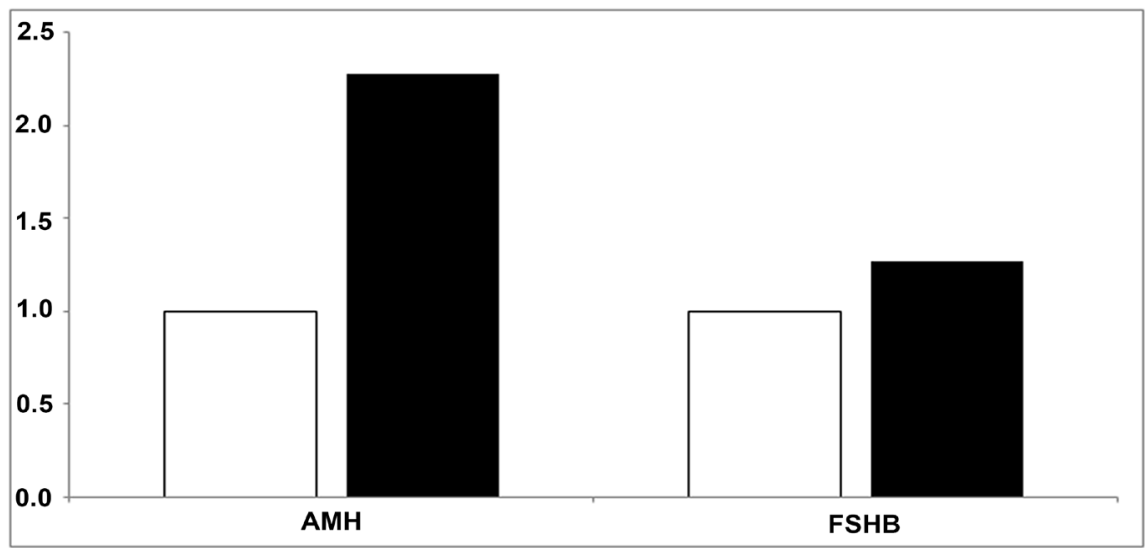

Figure 7. Stimulation of oocytes production.

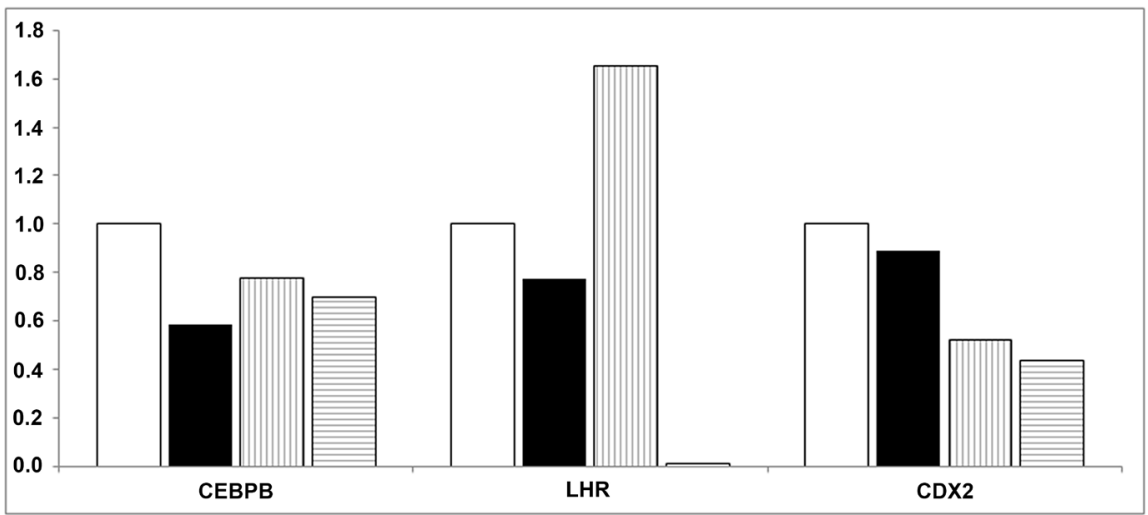

Figure 8. Gene analyses.

LHR expression shows a peculiar pattern; it was slightly down regulated in group 2 (0.8 times compared to the control), completely switched off in group 4, and upregulated in group 3 (1.6 times the control). The expression of CDX2 was down regulated in all the three groups, to a minor extent in group 2, about 0.9 times the control, and to a maximum extent in group 4 , where it was reduced to about 0.4 times that of the control.

\subsection{In Vivo Evaluation of Pregnancy Rate}

We verified if the above described genes expression regulation had some effect on the pregnancy rate in young and old mice (Figure 9 and Table 1).

The Group 2 old mice showed a significantly higher rate of pregnancy compared to control ( $80 \%$ vs $70 \%$ ), Group 2 and group 4 young mice show a comparable higher rate of pregnancy compared to control (100\% compared to $90 \%$ ). Group 3 of both young and old mice showed a lower rate of pregnancy compared to control group, in particular $30 \%$ vs $70 \%$ in old mice.

\section{Discussion}

We already published [14] our results on the effect of supplementation of carnitine with or without microelements on the fertility of 8 weeks old female mice. 


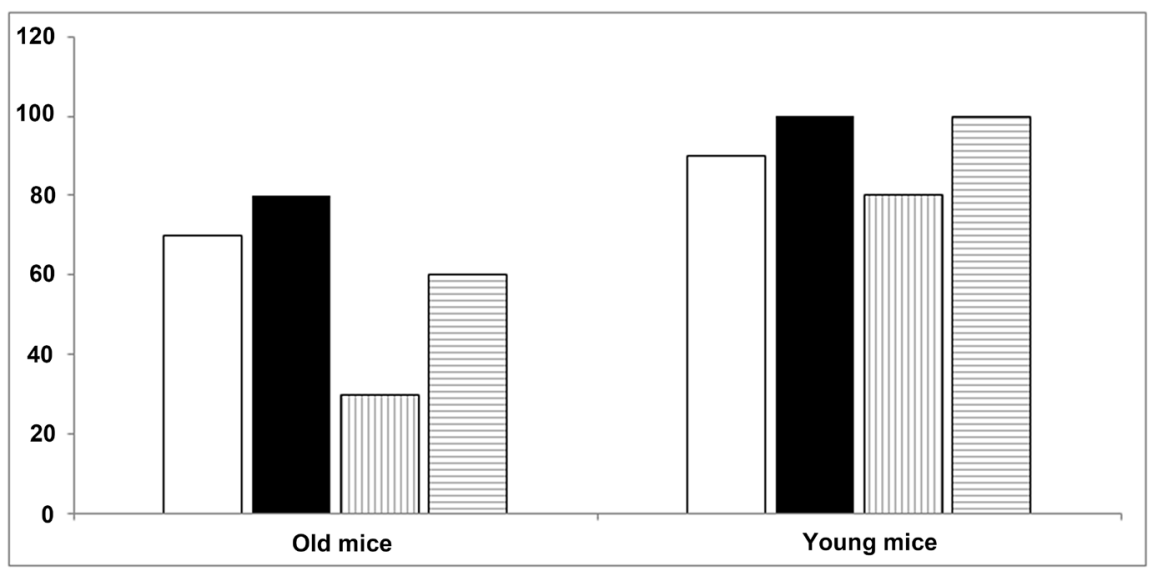

Figure 9. \% Pregnant.

In particular, we targeted the oocytes, evaluating the effect of supplementation on their quantity and quality, which resulted significantly improved in the groups assuming carnitines alone ad carnitine plus microelements. These results, prompted us to investigate both the causes and the consequences of the improvement in terms of quality and quantity of oocytes. In order to start to elucidate the molecular mechanism underling the oocytes status, we performed a preliminary evaluation of the expression of genes that are known to be in some way related to the female fertility. In particular: AMH (anti mullerian hormone) is produced by pre-antral follicles and is a biomarker of granulosa cell mass, and thus indirectly, of ovarian reserve [18]; FSHB (Follicle stimulating hormone Beta subunit) is part of FSH, hormone inducing the oocytes production; the expression of these two genes is thus directly related to an improvement of ovarian reserve and stimulation of oocytes production. CEBPB (CCAAT/enhancer binding protein beta) encodes a transcription factor important for the transcription of genes involved in immune and inflammatory responses, among other processes; LHR (Luteinizing hormone receptor) is the receptor of Luteinizing hormone (LH) by gonadotropic cells in the anterior pituitary gland and triggers ovulation and development of the corpus luteum [19]; CDX2 is a member of the caudal-related homeobox gene family, is related to placenta development and important in early embryogenesis [20] [21].

The expression of these last three genes is correlated to ovary aging and minor production of oocytes. Taking into account that this was a preliminary evaluation of the expression of the above mentioned genes, requiring following investigation, it seems quite clear a general positive effect of carnitine supplementation, mainly alone, in the direction of up regulation of the genes that favorite the fertility (AMH, FSH, FSHB), and down regulation of the genes that impair it, as also related with aging (CEBPB, LHR, CDX2).

Finally, we wanted to verify if all the results have correspondence with in vivo main indicator of fertility, i.e. the pregnancy rate. With the purpose to maximize the eventual effect of different supplementations, and explore a possible age ef- 
fect we administered the different supplementation protocols to two groups of mice differing in age young mice 5 weeks old, and old mice 8 weeks old. As expected, the global pregnancy rate was higher in all the four supplementation groups for young mice than old, but, among the groups of same age, again the groups receiving carnitine, alone or with macronutrients, showed a pregnancy rate significantly higher. Taking all together these results, we accumulated a strong evidence that the supplementation of carnitine has a beneficial effect on the female fertility, with an improvement of number and quality of oocytes, which is probably due or at least strongly connected with the up regulation of genes improving fertility and down regulation of genes which impair it, and with a final outcome in an increase of pregnancy rate.

An explanation could be that L-carnitine, which is essential in fatty acid metabolism, has been shown to prevent mitochondrial damage induced in the rat choroid plexus by medium chain fatty acids or by mitochondrial toxins. Key factors in the process of conception are the production of the ovum, the quality of the ovum and the state of the uterus. There is growing consensus that egg quality declines with age and in conditions of abnormal energy balance such as in polycystic ovary syndrome (PCOS). There is a critical period between ovulation and implantation when the egg has to rely exclusively on its own energy reserves contained within the zona pellucida. This energy comes from the fixed number of mitochondria present at moment of ovulation. If energy runs out the egg stops dividing and implantation is not achieved. In PCOS insulin resistance inhibits ovulation, impairs maturation of viable eggs, reduces egg quality and implantation. Restoring energy balance and providing adequate energy stores to the egg prior to ovulation would improve success of pregnancy. Further the oocyte genome quality could be improved by substances such as carnitines which improve genomic stability and therefore reduce chances of aneuploidy.

\section{Conclusion}

Environmental and stressful conditions can cause damage to the reproductive organs, eggs and general physiological processes of the body involved in the reproductive processes. The normal gene response may also be affected by metabolic dysfunctions. The metabolic compounds, such as carnitines, antioxidants and micronutrients may play an important role in fertility by effects on energy and free radical formation during cellular metabolic processes. Further improved mitochondrial function could provide adequate energy for successful conception as well as the period up to implantation in the uterus. Targeting ovum, tubal and uterus function may provide new approaches to improve fertility diseases however further experimental studies are needed in this field.

\section{Conflicts of Interest}

The authors declare no conflicts of interest regarding the publication of this paper. 


\section{References}

[1] Hakim, R.B., Gray, R.H. and Zacur, H. (1998) Alcohol and Caffeine Consumption and Decrease Fertility. Fertility and Sterility, 70, 632-637. https://doi.org/10.1016/S0015-0282(98)00257-X

[2] Gray, R.H. and Becker, S. (2000) Selected Topics in the Epidemiology of Reproductive Outcomes. Epidemiologic Reviews, 22, 71-75. https://doi.org/10.1093/oxfordjournals.epirev.a018027

[3] Virmani, M.A., et al. (2013) Food, Nutrigenomics and Neurodegeneration-Neuroprotection by What You Eat! Molecular Neurobiology, 48, 353-362. https://doi.org/10.1007/s12035-013-8498-3

[4] Virmani, M.A. and Angeli, A. (2013) Role of Nutrigenomics and Metabolic Markers in Evaluating Oxidative Stress and Inflammation Dysfunctions in Female Fertility. Abstract and Posters, Vitafoods, Geneva.

[5] Kim, C.S., et al. (1990) L-Carnitine Prevents Mitochondrial Damage Induced by Octanoid Acid in the Rat Choroid Plexus. Brain Research, 536, 335-338. https://doi.org/10.1016/0006-8993(90)90046-E

[6] Virmani, M.A., et al. (1995) Protective Actions of L-Carnitine and Acetyl-L-Carnitine on the Neurotoxicity Evoked by Mitochondrial Uncoupling or Inhibitors. Pharmacological Research, 32, 383-389. https://doi.org/10.1016/S1043-6618(05)80044-1

[7] Binienda, Z., et al. (1999) Protective Effect of L-Carnitine in the Neurotoxicity Induced by Mitochondrial Inhibitor 3-Nitrorpopionic Acid (3-NPA). Annals of the New York Academy of Sciences, 890, 173-178. https://doi.org/10.1111/j.1749-6632.1999.tb07992.x

[8] Matzuk, M.M. and Lamb, D.J. (2002) Genetic Dissection of Mammalian Fertility Pathways. Nature Cell Biology, 4, s41-49.

https://doi.org/10.1038/ncb-nm-fertilityS41

[9] Virant-Klun, I., Knez, K., Tomazevic, T. and Skutella, T. (2013) Gene Expression Profiling of Human Oocytes Developed and Matured In Vivo or In Vitro. BioMed Research International, 2013, Article ID: 879489. https://doi.org/10.1155/2013/879489

[10] Mansour, G., Abdelrazik, H., Sharma, R.K., Radwan, E., Falcone, T. and Agarwal, A. (2009) L-Carnitine Supplementation Reduces Oocyte Cytoskeleton Damage and Embryo Apoptosis Induced by Incubation in Peritoneal Fluid from Patients with Endometriosis. Fertility and Sterility, 91, 2079-2086. https://doi.org/10.1016/j.fertnstert.2008.02.097

[11] Virmani, M.A., Krismanovic, L.Z., Stojilkovic, S.S. and Catt, K.J. (1991) Stimulatory Effects of L-Acetylcarnitine on the Pituitary-Gonadal Axis in Female Rats. In: Adashi, E.Y. and Mancus, S., Eds., Major Advances in Human Female Reproduction, Raven Press, 73, 291-296.

[12] Debbie, M., Frida, E., Isabelle, L., Stephanie, B., Timur, G. and Yves, M. (2012) Carnitine Content in the Follicular Fluid and Expression of the Enzymes Involved in Beta-Oxidation in Oocyte and Cumulus Cells. Journal of Assisted Reproduction and Genetics, 29, 1221-1225. https://doi.org/10.1007/s10815-012-9855-2

[13] Dunning, K.R., Cashman, K., Russel, D.L., Thompson, J.G., Norman, R.J. and Robker, R.L. (2010) Beta-Oxidation Is Essential for Mouse Oocyte Development Competence and Early Embryo Development. Biology of Reproduction, 83, 909-918. https://doi.org/10.1095/biolreprod.110.084145

[14] Virmani, M.A., Zerelli, S., Vitullo, P. and Cossetti, C. (2015) Effect of Nutrients on 
Ovulation and Oocytes Quality in Mice. Giornale Italiano di Ostetricia e Ginecologia, 37, 212-214.

[15] Virmani, A., Diedenhofen, A. and Zerelli, S. (2014) Mitochondriotropic Compounds in Energy, Oxidative Stress and Inflammation: Role in Reproductive Health, Fertility and Successful Pregnancy. Giornale Italiano di Ostetricia e Ginecologia, 36, 293-296.

[16] Vitullo, P., Sciamanna, I., Baiocchi, M., Sinibaldi-Vallebona, P. and Spadafora, C. (2012) LINE-1 Retrotransposon Copies Are Amplified during Murine Early Embryo Development. Molecular Reproduction and Development, 79, 118-127. https://doi.org/10.1002/mrd.22003

[17] Meng, R. (2018) Relative Quantification: Data Management and Analysis Settings. Bio-Rad Laboratories Gene Expression Division.

http://www.bio-rad.com/genomics/pcrsupport

[18] Greene, A.D., Patounakis, G. and Segars, J.H. (2014) Genetic Associations with Diminished Ovarian Reserve: A Systematic Review of the Literature. Journal of Assisted Reproduction and Genetics, 31, 935-946.

https://doi.org/10.1007/s10815-014-0257-5

[19] Yding Andersen, C. (2017) Inhibin-B Secretion and FSH Isoform Distribution May Play an Integral Part of Follicular Selection in the Natural Menstrual Cycle. Molecular Human Reproduction, 23, 16-24. https://doi.org/10.1093/molehr/gaw070

[20] Beck, F., Erler, T., Russell, A. and James, R. (1995) Expression of Cdx-2 in the Mouse Embryo and Placenta: Possible Role in Patterning of the Extra-Embryonic Membranes. Developmental Dynamics, 204, 219-227.

https://doi.org/10.1002/aja.1002040302

[21] Strumpf, D., Mao, C.-A., Yamanaka, Y., Ralston, A., Chawengsaksophak, K., Beck, F. and Rossant, J. (2005) Cdx2 Is Required for Correct Cell Fate Specification and Differentiation of Trophectoderm in the Mouse Blastocyst. Development, 132, 2093-2102. https://doi.org/10.1242/dev.01801 